74 patients. 55/74 (74,3\%) patients experienced more than one hospitalization. In the majority of the hospitalizations $(119 / 285,41,7 \%)$, the cause of hospitalization was directly attributable to the disease itself, while the second cause of hospitalization was the infections $(26 / 285,9,1 \%)$. In $10 / 103$ patients $(9,7 \%)$, an end stage renal disease was recorded as event. The presence of at least one positivity for ANCA antibodies was documented in $76 / 103$ patients $(73,8 \%)$, mainly in patients carrying GPA. Globally, the presence of ANCA antibody seems to be associated with greater likelihood of an event ( $p=0,07$, log-rank test). The first event occurred in $50 \%$ of ANCA-positive patients within 180 days from diagnosis, while in $50 \%$ of ANCA negative patients in 859 days. 6 out of the 7 deaths occurred in ANCA positive patients.

Conclusion: the rate of hospitalization in AAV is very high confirming the high health care burden of illness. The disease itself is often the cause of the hospitalization, as well as the infectious complication, highlighting the need for more effective treatments, and glucocorticoid sparing therapies. ANCA antibody may represent a biomarker of a more serious disease.

Disclosure of Interests: Luca Quartuccio Consultant of: Abbvie, Bristol, Speakers bureau: Abbvie, Pfizer, Elena Treppo: None declared, Salvatore De Vita Consultant of: Roche, GSK, Speakers bureau: Roche, GSK, Novartis, Francesca Valent: None declared DOI: 10.1136/annrheumdis-2020-eular.2567

\begin{tabular}{|l|l}
\hline AB0521 & COST OF ILLNESS OF ANCA-ASSOCIATED \\
VASCULITIS IN ITALY: DATA LINKAGE ANALYSIS \\
OF MULTIPLE CLINICAL AND ADMINISTRATIVE \\
DATABASES IN THE PROVINCE OF UDINE, ITALY
\end{tabular}

L. Quartuccio ${ }^{1}$, E. Treppo ${ }^{1}$, S. De Vita ${ }^{1}$, F. Valent ${ }^{2} .{ }^{1}$ Clinic of Rheumatology, Department of Medicine, Academic Hospital "Santa Maria della Misericordia", ASUI, Udine, Italy, Udine, Italy; ${ }^{2}$ Institute of Epidemiology, Academic Hospital "Santa Maria della Misericordia”, ASUI, Udine, Italy, Udine, Italy

Background: ANCA-associated vasculitides (AAV) are a group of systemic vasculitis carrying a high risk of hospitalization because the multiorgan involvement, the acute nature of some clinical manifestations, the chronic but very disabling course of some other manifestations and finally the risk of severe infections due to chronic glucocorticoid and immunosuppressor administration. However, data on cost of illness due to AAV are lacking.

Objectives: to estimate the cost of illness in patients suffering from AAV in the province of Udine (about 500,000 inhabitants), Friuli Venezia Giulia (FVG), Italy, from year 2010 to 2018 .

Methods: integration of the information coming from many administrative databases were used to this end. The Regional Health Information System of FVG was used as the source of information for this retrospective cohort study. The system covers the entire regional population and includes various electronic health administrative databases that can be linked with one another on an individual basis through a unique encrypted identifier. In particular, the following databases were matched: the database of the health care beneficiaries (including demographic information and the residential history of all of the subjects living in FVG), the hospital discharge database, the database of exemptions from medical charges, the database of the laboratories. The population under study was selected based on the following inclusion criteria: patients were residents in the province of Udine and they had to carry the exemption code for AAV, including GPA, or EGPA, or MPA. This population was observed from 2010 to 2018.

Results: 57 patients (201 patient-years) with AAV were identified. They were ANCA-positive in 44/57 (77\%). GPA, EGPA and MPA was diagnosed in 18 $(31,6 \%), 15(26,3 \%), 11(19,3 \%)$ patients, respectively. The mean age at diagnosis was $54,5(17,5)$ years. The disease itself was the main cause of hospitalization in almost half of the hospital discharges (60/126, 47,6\%). Four patients died during the observation period due to vasculitis itself (1), pneumonia (2), or haematological malignancy (1). Time to the first event (death or hospitalization) was significantly higher in ANCA-negative AAV patients than in ANCA-positive AAV patients ( $p=0,03$, Log-Rank test), ANCA-positive AAV patients having a three-times higher risk ( $\mathrm{HR} 3,3895 \% \mathrm{Cl} 1,13-10,08$, $p=0,03$ ). Total estimated cost was $€ 1,215,078$, corresponding to $€ 6,168$ patient-year. Costs for ANCA-positive AAV patients were much higher than those for ANCA-negative AAV patients ( $€ 1,115,253$ vs $€ 99,825$, and $€ 7058$ per person-year vs $€ 2,559$ per person-year, respectively). GPA and MPA showed the highest costs if compared to EGPA [GPA: $€ 239,168$ ( $€ 5199$ per person-year) vs MPA: $€ 281,502$ (€ 4771 per person-year) vs EGPA: $€ 214,287$ (2329 per person-year), respectively]. Costs for hospitalization were the highest [ $€ 734,957$ ( $€ 3731$ per person-year) vs other costs $€ 480,121$ ( $€ 2437$ per person-year)].

Conclusion: costs for AAV are very high, confirming the high health care burden of this illness. Management of ANCA-positive patients rather than ANCA-negative patients was burdened by the highest costs. GPA and MPA showed the highest direct costs for hospitalization, which very frequently occurred due to the vasculitis itself.

Disclosure of Interests: Luca Quartuccio Consultant of: Abbvie, Bristol, Speakers bureau: Abbvie, Pfizer, Elena Treppo: None declared, Salvatore De Vita Consultant of: Roche, GSK, Speakers bureau: Roche, GSK, Novartis, Francesca Valent: None declared

DOI: 10.1136/annrheumdis-2020-eular.2585

\section{AB0522 GENDER DIFFERENCES IN GIANT CELLS ARTERITIS: ANALYSIS OF A MONOCENTRIC COHORT OF 100 PATIENTS.}

F. Regola ${ }^{1}$, A. Tincani ${ }^{1}$, F. Franceschini ${ }^{1}$, P. Toniati ${ }^{1} .{ }^{1}$ ASST Spedali Civili and University of Brescia, Rheumatology and Clinical Immunology Unit, Brescia, Italy

Background: Giant Cells Arteritis (GCA) is the most common primary vasculitis in adults and usually occurs in patients older than 50 years. Epidemiological studies shown a higher prevalence of the disease in women compared to man. However, differences in clinical presentation between men and women have not been demonstrated, even if some distinctions have been suggested $(1,2)$.

Objectives: The purpose of the present study is to analyze differences in the clinical presentation of GCA according to sex.

Methods: We collected retrospectively clinical data of a monocentric cohort of 100 consecutive GCA patients. Mann Whitney test was used to compare continuous variables, while Chi-square test and Fisher's exact test were applied for comparison between qualitative variables.

Results: One-hundred patients with a clinical diagnosis of GCA were enrolled in the study ( 68 women, 32 men). In all patients the diagnosis of vasculitis was histologically and/or radiologically confirmed. Main clinical data are reported in the table.

Patients were classified according to vascular involvement in three groups: temporal arteritis (C-GCA), extracranial large vessel vasculitis (LV-GCA) and both cranial and extracranial vasculitis (LV-C-GCA). No significant differences in vascular distribution of the disease were found according to sex, even if large vessel involvement seems to be more frequent in women (43\% vs $28 \%$; : ns).

Male and female patients presented at diagnosis a similar clinical picture, with the same frequency of systemic symptoms (fever, fatigue, weight loss), polymyalgia rheumatica, visual symptoms and claudication. However, male patients complained more often temporal headache $(90 \%$ vs $71 \%$, p: 0.01$)$, even no significant differences were found in the incidence of pathological findings at temporal artery physical examination (38\% vs 32\%; p: ns) and biopsy (59\% vs $50 \%$ ) On the contrary, in female patients a longer time to diagnosis was recorded (8 (2-49 vs 4 (0-35) months; $p: 0.01)$.

Conclusion: In our cohort of GCA patients, clinical presentation was similar in male and female patients, with no significant differences in clinical, radiological and laboratory findings. However, male patients presented more often temporal headache, the most typical symptom of GCA, and this could explain a shorter time to diagnosis, if compared to female.

References:

[1] Sturm A et al. Clin Exp Rheum, 2016

[2] Nir-Paz R et al. J of Rheum, 2002

\begin{tabular}{lccc}
\hline & $\begin{array}{c}\text { All GCA } \\
(\mathrm{n}: 100)\end{array}$ & WOMAN (n:68) & $\begin{array}{c}\text { MAN } \\
(\mathrm{n}: 32)\end{array}$ \\
\hline Age: median $\left(10^{\text {th }}-90^{\text {th }}\right.$ percentile) & $76(62-80)$ & $73(60-83)$ & $76(62-80)$ \\
Time to diagnosis (weeks) ${ }^{*}$ & $8(1-42)$ & $8(2-49)$ & $4(0-35)$ \\
C-GCA & $61(61 \%)$ & $38(56 \%)$ & $23(72 \%)$ \\
LV-C-GCA & $16(16 \%)$ & $13(20 \%)$ & $3(9 \%)$ \\
LV-GCA & $23(23 \%)$ & $17(25 \%)$ & $6(20 \%)$ \\
New temporal headache* & $77(77 \%)$ & $48(71 \%)$ & $29(90 \%)$ \\
Visual symptoms & $39(39 \%)$ & $24(35 \%)$ & $15(47 \%)$ \\
Jaw or tongue claudication & $35(35 \%)$ & $23(34 \%)$ & $12(38 \%)$ \\
Fever & $39(39 \%)$ & $24(35 \%)$ & $15(47 \%)$ \\
Fatigue & $71(71 \%)$ & $48(71 \%)$ & $23(72 \%)$ \\
Weight loss & $49(49 \%)$ & $34(50 \%)$ & $15(47 \%)$ \\
Polymyalgia rheumatica & $43(43 \%)$ & $33(49 \%)$ & $10(31 \%)$ \\
Arm or leg claudication & $5(5 \%)$ & $2(3 \%)$ & $3(9 \%)$ \\
Positive temporal artery biopsy & $53(53 \%)$ & $34(50 \%)$ & $19(59 \%)$ \\
Pathological findings at temporal artery physical & $34(34 \%)$ & $22(32 \%)$ & $12(38 \%)$ \\
$\quad$ examination & & & \\
CRP (C-reactive protein) & $83(22-160)$ & $89(21-159)$ & 78 \\
& & & $(23-155)$ \\
ESR (erythrocyte sedimentation rate) & $72(34-109)$ & $79(37-113)$ & 54 \\
FDG-PET activity throughout aorta & $32(32 \%)$ & $25(37 \%)$ & $(32-100)$ \\
& & & $7(22 \%)$ \\
\hline
\end{tabular}

${ }^{*}: p \leq 0.05$ 
Disclosure of Interests: None declared

DOI: 10.1136/annrheumdis-2020-eular.6524

\section{AB0523 TAKAYASU ARTERITIS AND SACROILIITIS: A CASE- CONTROL STUDY}

F. Regola ${ }^{1}$, G. Bosio ${ }^{2}$, F. Franceschini ${ }^{1}$, P. Toniati ${ }^{1}{ }^{1}$ ASST Spedali Civili and University of Brescia, Rheumatology and Clinical Immunology Unit, Brescia, Italy; ${ }^{2}$ ASST Spedali Civili of Brescia, Nuclear Medicine Unit, Brescia, Italy

Background: A possible shared immunopathogenesis between Spondyloarthritis (SpA) and Takayasu Arteritis (TA) has been hypothesized and some clinical cases about SpA in TA patients have been reported (1). In clinical practice the diagnosis of sacroiliitis may be performed by X-ray, Computed Tomography (CT) or Magnetic Resonance Imaging (MRI). In particular, CT findings of sacroiliitis include contour irregularities, joint space alterations, joint erosion, subcondral bone changes (osteoporosis or sclerosis), enthesitis, ankylosis. Meanwhile, TA patients performe routinely FDG-PET/CT scans for monitoring disease activity.

Objectives: This study aims to understand if there is an increased incidence of sacroilititis in TA patients.

Methods: We collected retrospectively imaging data from FDG-PET/CT scans of 28 TA patients and 28 controls, matched for sex and age. Controls were selected among patients performing FDG-PET/CT in our Nuclear Medicine Unit, excluding patients with bone tumors, bone metastasis and thyroid cancers. The majority of controls were affected by lymphoma in complete remission. An expert rheumatologist read the CT-scans of sacroiliac joints.

Results: No patients or controls demonstrated FDG-uptake in sacroiliac joints. In the control group we detected sacroiliac sclerosis in two cases: one due to degenerative changes, one to sacroiliitis (1/28, $4 \%$ ). In the TA group four patients presented CT alterations suggestive for sacroilitis: one bilateral erosion, one bilateral sclerosis, two monolateral sclerosis $(4 / 28,14 \%)$. One of these patients complained an inflammatory back pain.

Conclusion: In our cohort of TA patients we demonstrated an increased prevalence of sacroilitis, diagnosed by CT scan. Only one patient reported an inflammatory back pain, while three patients had radiological signs of previous sacroilitis. These findings highlight the importance of looking for spondyloarthropathy in TA patients even if asymptomatic.

References:

[1] Guzel Esen S, Joint Bone Spine, 2019

Disclosure of Interests: None declared

DOI: 10.1136/annrheumdis-2020-eular.3940

\section{AB0524 ANCA ASSOCIATED VASCULITIS IN GRAN CANARIA: THE IMPORTANCE OF THE INTERSTITIAL LUNG DISEASE}

F. J. Nóvoa Medina ${ }^{1}$, F. Rubiño $^{2}$, B. Tejera-Segura ${ }^{1}$, B. Romero Díaz ${ }^{3}$, S. Machín García ${ }^{1}$, I. Rua-Figueroa ${ }^{2} .{ }^{1}$ Complejo Hospitalario Universitario Insular Materno Infantil de Gran Canaria, Rheumatology, Las Palmas de Gran Canaria, Spain; ${ }^{2}$ Hospital de Gran Canaria "Dr. Negrín", Rheumatology, Las Palmas de Gran Canaria, Spain; ${ }^{3}$ Complejo Hospitalario Universitario Insular Materno Infantil de Gran Canaria, Radiology, Las Palmas de Gran Canaria, Spain

Background: ANCA-associated vasculitis (AVV) are a heterogeneous group of systemic diseases that needs a better knowledge and approach due to the high mortality it presents.

Objectives: Describe the clinical characteristics of patients with AAV assessed by the Rheumatology services in two university hospitals in Gran Canaria in the last decade, as well as clinical differences between the AAV subtypes.

Methods: Characteristics of 34 patients diagnosed with AAV between January 2011 - December 2018 were collected retrospectively. The patients met ACR classification criteria and consensus criteria from Chapell Hill-2012. Variables are compared using the $x^{2}$ test for dichotomous variables or the t-Student test for continuos variables. For non-continuous variable, Mann-Whitney $U$ or a logarithmic transformation was used

Results: $21(61.7 \%)$ patients were women. We found 14 granulomatosis with polyangeiitis (GPA 41.2\%), 10 microscopic polyangeiitis (MPA) and 10 eosinophilic granulomatosis with polyangeiitis (EGP) $(29.4 \%)$. They presented an average follow-up time $( \pm S D)$ of 46.3 months $( \pm 26.8)$. Patients with MPA presented an older age at diagnosis and a higher proportion were diagnosed with age $>65$ years $(p=0.003)$.

The mean $( \pm \mathrm{SD})$ of the BVAS index of activity at diagnosis was 15,7 $( \pm 7.9)$. $80,5 \%$ of the patients presented positivity against ANCA: $34,4 \%$ c-ANCA and $65.5 \%$ (Clinical manifestations in Table 1).
TABLE 1. INITIAL CLINICAL MANIFESTATIONS

\begin{tabular}{|c|c|c|c|c|c|}
\hline & $\begin{array}{c}\text { ALL THE } \\
\text { PATIENTS }(n=34)\end{array}$ & $\begin{array}{c}\text { GPA } \\
(n=14)\end{array}$ & $\begin{array}{l}\text { MPA } \\
(n=10)\end{array}$ & $\begin{array}{c}E G P \\
(n=10)\end{array}$ & $\begin{array}{c}\mathrm{p} \\
\text { value }\end{array}$ \\
\hline Otolaryngological involvement & & $13(92.9 \%)$ & $2(20 \%)$ & $6(60 \%)$ & ) 0.001 \\
\hline Interstitial lung disease & $5(14.7 \%)$ & 0 & $5(50 \%)$ & 0 & \\
\hline Renal involvement & & & & & 0.027 \\
\hline - Reno-pulmonary syndrome & $6(17,6 \%)$ & $3(21.4 \%)$ & $2(20 \%)$ & $1(10 \%)$ & \\
\hline $\begin{array}{l}\text { - Renal Involvement } \\
\text { - glomerulonephritis }\end{array}$ & $10(29.4 \%)$ & $3(21.4 \%)$ & $6(60 \%)$ & $1(10 \%)$ & \\
\hline - Basal proteinuria $>1 \mathrm{gr} / 24 \mathrm{hs}$ & $13(38.2 \%)$ & $4(28.5 \%)$ & $7(70 \%)$ & $1(10 \%)$ & \\
\hline $\begin{array}{l}\text { Alveolar pulmonary hemorrhage not } \\
\text { associated with renal involvement }\end{array}$ & $2(5.8 \%)$ & 0 & $1(10 \%)$ & $1(10 \%)$ & \\
\hline $\begin{array}{l}\text { Manifestations Peripheral Nervous } \\
\text { System }\end{array}$ & $10(29.4 \%)$ & $2(14.2 \%)$ & $4(40 \%)$ & $4(40 \%)$ & \\
\hline Cardiomyopathy & $3(8.8 \%)$ & 0 & 0 & $3(30 \%)$ & \\
\hline $\begin{array}{l}\text { Eye involvement (scleritis/conjunctivitis/ } \\
\text { keratitis/uveitis) }\end{array}$ & $6(17.6 \%)$ & $6(42.8 \%)$ & 0 & 0 & 0.01 \\
\hline
\end{tabular}

$21(61.7 \%)$ patients received cyclophosphamide and $3(8.8 \%)$ patients received rituximab as induction treatment. Azathioprine was the most com monly used maintenance treatment $(41,1 \%) .16(47 \%)$ patients had renal involvement. An improvement in proteinuria was observed, both in GPA $(p=0.008)$ and in MPA $(p=0,03)$ (Renal outcomes in Table 2). No patient received kidney transplant.

TABLE 2. RENAL OUTCOMES

\begin{tabular}{lccc}
\hline & GPA $(n=6)$ & MPA $(n=8)$ & p VALUE \\
\hline Basal serum creatinine, mean (SD), mg/dl & $2.07(1.1)$ & $3.08(2.06)$ & 0.3934 \\
Basal proteinuria, mean (SD), mg/dl & $2264(1391.5)$ & $2731.2(1334.7)$ & 0.8348 \\
Last serum Creatinine, mean (SD), mg/dl & $2,2(1.4)$ & $2,1(1.5)$ & 0.5577 \\
Last proteinuria, mean (SD), mg/dl & $485(457.9)$ & $326(110.4)$ & 0.4704 \\
\hline
\end{tabular}

Interestingly, 5 patients $(14.7 \%)$, all of them MPA, presented interstitial lung disease (ILD), 3 of them (60\%) prior to systemic involvement $(9,10$ and 82 months). 3 patients had an usual interstitial pneumonitis (UIP) pattern, none had a non-specific interstitial pneumonia (NSIP) pattern and two had other patterns.

15 patients had 17 relapses. Five (14.7\%) patients had serious infections. Eight (23.5\%) patients died: 4 due to progression of ILD, 2 due to vasculitis manifestations.

Conclusion: ILD can be considered a relatively frequent manifestation of this group of diseases. A high percentage of patients had recurrences. Mortality remains high in AAV and in our series ILD is a frequent cause of death.

Disclosure of Interests: Francisco Javier Nóvoa Medina Speakers bureau: have been paid as a speaker for a few medical talks, Francisco Rubiño: None declared, Beatriz Tejera-Segura Speakers bureau: I have been paid as a speaker for a few medical talks, Beatriz Romero Díaz: None declared, Sergio Machín García: None declared, Iñigo Rua-Figueroa: None declared

DOI: 10.1136/annrheumdis-2020-eular.3268

\begin{tabular}{|l|l|}
\hline AB0525 & NATURAL HISTORY OF CRYOGLOBULINEMIA \\
& FROM 2000 TO 2018 FROM THE LABORATORY \\
& POINT OF VIEW: AN ANALYSIS OF CRYOGLOBULIN \\
& CHARACTERISTICS IN A SINGLE CENTER.
\end{tabular}

G. Sandri ${ }^{1}$, A. Spinella ${ }^{1}$, P. Natali ${ }^{2}$, D. Debbia ${ }^{2}$, D. Campioli ${ }^{2}$, A. Bari ${ }^{3}$ G. Amati ${ }^{1}$, G. Galassi ${ }^{4}$, M. Mazzoli ${ }^{4}$, G. Alfano ${ }^{5}$, F. Fontana ${ }^{5}$, T. Trenti ${ }^{2}$, M. T. Mascia ${ }^{1} .{ }^{1}$ Chair and Complex Operational Unit of Rheumatology, University of Modena and Reggio Emilia, Modena, Italy; ${ }^{2}$ Department of Laboratory Medicine and Anatomical Pathology, AOU and AUSL of Modena, Modena, Italy; ${ }^{3}$ Complex Structure of Oncology and Hematology, AOU of Modena, Modena, Italy; ${ }^{4}$ Department of Neuroscience, AOU of Modena, Modena, Italy; ${ }^{5}$ Complex Structure of Nephrology and Dialysis, AOU of Modena, Modena, Italy

Background: Big data refers to large amounts of information. With today's ever-improving technologies created by the automation and digitization, it becomes easier to convert data into relevant information, which can be used to provide better patient management, especially when it occurs a rare condition such as cryoglobulinemia (CRG).

CRG is due to an immunoglobulins (Ig) that precipitate at low temperatures. There are 3 types of CRG: type I: monoclonal Ig; type II: monoclonal Ig + polyclonal Ig; type III: 2 polyclonal Ig. 